Ann. Zootech., I973, 22 (I), 6I-72.

\title{
VALEUR COMPARÉE DES PROTÉINES DE LEVURE SULFITIQUE ET DE LACTOSÉRUM LEVURÉ DANS UNE RATION A BASE D'ORGE, CHEZ LE PORC EN CROISSANCE-FINITION. EFFET D'UNE SUPPLÉMENTATION EN DL-MÉTHIONINE
}

\author{
C. FÉVRIER, F. COLOMER-ROCHER et B. SÈVE \\ Station de Recherches sur l'Élevage des Porcs, \\ Centre national de Recherches zootechniques, I. N. R. A., \\ 78350 Jouy en Josas
}

\section{RÉSUMÉ}

On a comparé sur 72 porcs de race Large White de 25 à $90 \mathrm{~kg}$, l'efficacité de trois sources azotées : levure sulfitique, lactosérum levuré et tourteau de soja " 44 ", dans des régimes à base d'orge, isoprotidiques ( I3 p. Ioo $\mathrm{N} \times 6,25)$, isocalorique $(4300 \mathrm{kcal} / \mathrm{kg}$ ) et isocellulosiques ( 10 p. 1 oo de cellulose brute), supplémentés ou non par o, I p. Ioo de DL-méthionine de synthèse. Les régimes non supplémentés entraînent des performances zootechniques identiques chez les animaux. L'effet favorable de la supplémentation n'atteint pas le seuil de signification statistique dans les régimes à base de levure sulfitique et de tourteau de soja. La supplémentation en méthionine du régime lactosérum levuré entraîne, par contre, une réduction significative de la vitesse de croissance, consécutive à un excès d'acides aminés soufrés dans la ration. En revanche, le coefficient de rétention azotée est significativement amélioré par la supplémentation en acides aminés soufrés, dans le cas du régime à base de levure sulfitique. Enfin, la qualité des carcasses reste constante, quelle que soit la source de protéines utilisées ou le taux d'acides aminés soufrés du régime.

Ces résultats montrent que dans les conditions de l'expérience, la levure sulfitique et le lactosérum levuré peuvent se substituer sans inconvénient au tourteau de soja dans l'aliment du Porc en croissance-finition.

\section{INTRODUCTION}

La production de protéines de levures présente un intérêt de plus en plus reconnu, puisqu'elle peut être réalisée sur des déchets organiques industriels contribuant ainsi à leur valorisation et à la lutte contre la pollution des eaux. Mais ces protéines 
doivent être obtenues dans des conditions économiques telles que leur utilisation dans 1'alimentation animale soit compétitive au regard des protéines actuellement disponibles, et notamment vis-à-vis du tourteau de soja.

D'après leur composition chimique, les levures aliments, présentent un déficit primaire en acides aminés soufrés, ce qui a été confirmé expérimentalement par de nombreux auteurs, et chez différentes espèces animales : volailles, pores et rats

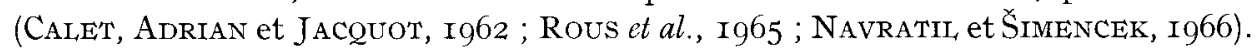
Au cours de l'une de nos expériences précédentes sur Porcs, nous avons observé, que la rétention azotée obtenue avec le régime orge-soja était supérieure, à apport azoté égal, à celle de régimes renfermant des levures (COLOMER-ROCHER et FÉVRIÊR I968). Par la suite, la distribution des mêmes régimes à des Rats nous a permis de montrer que setule la méthionine permettait d'améliorer la rétention azotée du régime orge-levure sulfitique (COLOMER-ROCHER et FÉVRIER, I97I).

L'objet de la présente étude est done de vérifier l'effet bénéfique de l'addition de méthionine à la levure, en se basant sur les besoins définis par RÉRAT et Lougnon, ( I 666), dans le cas de porcs en croissance-finition, recevant une alimentation rationnée. Une première expérience a porté sur des lots de porcs alimentés avec les trois régimes précédemment étudiés et une seconde a été réalisée en cage à métabolisme, avec la levure sulfitique.

\section{MÉTHODES EXPÉRIMENTALES}

\section{A. - Expérience en lots}

Soixante-douze porcs de race Large White, de 25 à $30 \mathrm{~kg}$, ont été répartis en six lots de 6 femelles et 6 mâles castrés, suivant un dispositif en blocs complets constitués sur la base d'un poids et d'un âge équivalents lors de la mise en lot. Le schéma expérimental était le suivant :

\begin{tabular}{|c|c|c|c|c|c|c|}
\hline Régime & \multicolumn{2}{|c|}{ Orge-soja } & \multicolumn{2}{|c|}{$\begin{array}{l}\text { Orge-levure } \\
\text { sulfitique }\end{array}$} & \multicolumn{2}{|c|}{$\begin{array}{c}\text { Orge-lactosérum } \\
\text { levuré }\end{array}$} \\
\hline $\begin{array}{l}\text { Supplémentation } \\
\text { DL-méthionine }\end{array}$ & 0 & + & 0 & + & 0 & + \\
\hline
\end{tabular}

La composition détaillée des régimes est rapportée au tableau $\mathbf{I}$. Les protéines à tester (levures et soja) fournissent $48 \mathrm{p}$. Ioo des matières azotées totales de la ration. On a tenu compte dans la composition des mélanges minéraux, de différences de teneur en $\mathrm{P}$, Ca et $\mathrm{Na}$.

L'analyse chimique (tabl. 2) montre que les régimes sont isocaloriques (4 $300 \mathrm{kcal} / \mathrm{kg}$ ), isoprotidiques ( 13 p. Ioo $\mathrm{N} \times 6,25$ ) et isocellulosiques ( 10 p. Ioo de cellulose brute).

Le taux de supplémentation en DL-méthionine est fixé à o, I p. I oo de la ration, permettant ainsi d'inverser l'ordre des facteurs limitants théoriques, acides aminés soufrés et lysine, dans chacun des régimes (tab1. 3).

Les animaux étaient élevés en loge collective et alimentés individuellement deux fois par jour, sous forme humide, suivant un plan de rationnement rapporté au tableau 4 . Les quantités offertes augmentaient jusqu'à $3,0 \mathrm{~kg}$ d'aliment, niveau atteint à $70 \mathrm{~kg}$ de poids vif. Les animaux ont été pesés chaque semaine et la matière sèche des refus déterminée. 
TABLEAU I. - Composition des régimes (p. Ioo)

\begin{tabular}{|c|c|c|c|}
\hline Régimes (p. 100) & Soja & $\begin{array}{l}\text { Levure } \\
\text { sulfitique }\end{array}$ & $\begin{array}{c}\text { Lactosérum } \\
\text { levuré }\end{array}$ \\
\hline Orge $\ldots \ldots \ldots \ldots \ldots \ldots$ & 59,00 & 59,00 & 59,00 \\
\hline Levure sulfitique ....... & & 13,00 & \\
\hline Tourteau de soja " 44 " cuit. & 16,20 & - & - \\
\hline Lactosérum levuré $\ldots . .$. & - & - & 25,00 \\
\hline Cellulose fine $\ldots \ldots \ldots \ldots$ & 4,00 & 5,00 & 5,00 \\
\hline Amidon de maïs........ & 11,98 & 14,18 & 5,26 \\
\hline Huile d'arachide $\ldots \ldots \ldots$ & 1,00 & 1,00 & 1,00 \\
\hline Mélange minéral I $(a)$. & 3,82 & - & - \\
\hline$-\quad-\quad$ II. & - & 3,82 & - \\
\hline$\longrightarrow \quad-\quad \mathrm{III} \longrightarrow$. & - & - & 0,74 \\
\hline Mélange vitaminique $(c)$. & 4,00 & 4,00 & 4,00 \\
\hline $\begin{array}{l}\text { DL-méthionine } \\
\text { (régimes supplémentés) }\end{array}$ & 0,10 & 0,10 & 0,10 \\
\hline $\begin{array}{c}\text { (a) Mélanges minéraux } \\
\text { (p. 100) }\end{array}$ & 1 & 2 & 3 \\
\hline Phosphate bicalcique .... & 48,43 & 55,76 & 51,35 \\
\hline Carbonate de calcium ... & 23,30 & 15,97 & - \\
\hline Chlorure de sodium ..... & 18,85 & 18,85 & 一 \\
\hline Oligo-éléments $(b) \ldots \ldots$. & 9,42 & 9,42 & 48,65 \\
\hline
\end{tabular}

(b) Oligo-éléments :

Carbonate de magnésium..... $\quad 83,33$

Sulfate ferreux .......... 8,33

Sulfate de manganèse $\ldots \ldots \ldots . \quad 2,50$

Sulfate de cuivre $\ldots \ldots \ldots \ldots \ldots \quad 0,84$

Sulfate de cobalt ......... 0,42

Sulfate de $Z_{n} \ldots \ldots \ldots \ldots \ldots . \quad 4,16$

Iodure de potassium ....... 0,42

(c) Vitamines :

Vit. A : $125000 \mathrm{UI}$

Riboflavine $50 \mathrm{mg}$

Choline $25 \mathrm{mg}$

Orge q.s.p. $1 \mathrm{~kg}$
Vit. $D_{3}: 12500 \mathrm{UI}$

A. pantothénique $125 \mathrm{mg}$

Vit. $\mathrm{B}_{12} \quad 0,25 \mathrm{mg}$

TABLEAU 2. - Composition chimique des régimes

\begin{tabular}{|c|c|c|c|}
\hline Régime & Soja & $\begin{array}{l}\text { Levure } \\
\text { sulfitique }\end{array}$ & $\begin{array}{l}\text { Lactosérum } \\
\text { levuré }\end{array}$ \\
\hline Matière sèche & 86,5 & 87,0 & 87,4 \\
\hline Matières minérales ......... & 5,2 & 5,2 & 6,5 \\
\hline Matières grasses .......... & 2,6 & 2,2 & 2,6 \\
\hline Matières azotées $(\mathrm{N} \times 6,25)$. & 13,1 & 13,2 & 13,2 \\
\hline Cellulose brute (calculée) .... & 10,0 & 10,2 & 10,0 \\
\hline Énergie brute (kcal/kg MS). . & 4289 & 4332 & 4303 \\
\hline $\mathrm{Ca}$ (calculé) $\ldots \ldots \ldots \ldots$ & 0,8 & 0,8 & 0,8 \\
\hline $\mathrm{P}$ (calculé) $\ldots$ & 0,75 & 0,75 & 0,75 \\
\hline
\end{tabular}


Après l'abattage à $90 \mathrm{~kg}$, les carcasses ont été traitées suivant la découpe parisienne normalisée, après un ressuyage de $24 \mathrm{~h}$.

L'analyse statistique des résultats a été effectuée suivant un schéma en blocs complets à deux facteurs contrôlés, sexe et régime, permettant la mise en évidence des interactions (CocHRAN et Cox, 1957).

\section{TABLEAU 3}

Calcul du pourcentage de déficit des régimes relativement aux besoins théoriques du porc en lysine et méthionine (RÉrat et Lougnon, I966; Pion, 1966-1967)

\begin{tabular}{|c|c|c|c|c|c|}
\hline \multicolumn{3}{|c|}{ Régime } & Soja & $\begin{array}{l}\text { Levure } \\
\text { sulfitique }\end{array}$ & $\begin{array}{c}\text { Lactosérum } \\
\text { levuré }\end{array}$ \\
\hline $\begin{array}{l}\text { Poids vif } \\
\qquad(\mathrm{kg})\end{array}$ & Acides aminés & $\begin{array}{c}\text { Normes } \\
(\mathrm{g} / \mathrm{j})\end{array}$ & \multicolumn{3}{|c|}{ P. 100 déficit/normes } \\
\hline 20 & $\begin{array}{l}\text { Lysine ................ } \\
\text { Méthionine }+ \text { cystine ..... } \\
\text { Méthionine après supplém. . }\end{array}$ & $\begin{array}{l}8,7 \\
6,1 \\
-\end{array}$ & $\begin{array}{r}20 \\
21 \\
4\end{array}$ & $\begin{array}{l}16 \\
29 \\
12\end{array}$ & $\begin{array}{r}11 \\
16 \\
0\end{array}$ \\
\hline 40 & $\begin{array}{l}\text { Lysine .............. } \\
\text { Méthionine }+ \text { cystine ..... } \\
\text { Méthionine après supplém. . }\end{array}$ & $\begin{array}{l}14 \\
10,9 \\
-\end{array}$ & $\begin{array}{r}17 \\
25 \\
9\end{array}$ & $\begin{array}{l}12 \\
32 \\
16\end{array}$ & $\begin{array}{r}7 \\
20 \\
5\end{array}$ \\
\hline 70 & $\begin{array}{l}\text { Lysine ................ } \\
\text { Méthionine }+ \text { cystine . . . } \\
\text { Méthionine après supplém. . }\end{array}$ & $\begin{array}{c}18,5 \\
1^{\prime}, 2 \\
-\end{array}$ & $\begin{array}{l}0 \\
1 \\
0\end{array}$ & $\begin{array}{r}0 \\
11 \\
0\end{array}$ & $\begin{array}{l}0 \\
0 \\
0\end{array}$ \\
\hline
\end{tabular}

TABLEAU 4

Rationnements utilisés

\begin{tabular}{|c|c|c|c|}
\hline Expérience & $\begin{array}{l}\text { COLOMER-RoCHER } \\
\text { et FÉvRIER (1968) }\end{array}$ & \multicolumn{2}{|c|}{ Expérience présente } \\
\hline Habitat & Cages à bilan & Cages à bilan & Loges \\
\hline Poids vif & \multicolumn{3}{|c|}{ Quantités d'aliment ( $\mathrm{g}$ MS/j) } \\
\hline 30 & 900 & 1100 & 1300 \\
\hline 40 & 1200 & 1300 & 1700 \\
\hline 50 & 1600 & - $^{*}$ & 2100 \\
\hline 60 & 1800 & 2000 & 2500 \\
\hline 70 & 1950 & $-^{*}$ & 2900 \\
\hline 80 & 2000 & $-*$ & 3000 \\
\hline & & & \\
\hline
\end{tabular}

* Animaux " hors expérience " rationnés de la même façon que les animaux en loges. 


\section{B. - Bilan azoté effectué avec le régime "levure sulfitique "}

Seuls les traitements correspondant à la levure sulfitique ont été utilisés sur deux lots de 4 porcs mâles castrés, de poids et d'âge comparables.

Placés en cage à métabolisme et alimentés suivant le rationnement du tableau 4 , les porcs ont subi une période préexpérimentale d'une semaine, puis deux périodes de collecte de six jours chacune, à un poids moyen de $35 \mathrm{~kg}$. Après un séjour, au sol, en porcherie, deux nouvelles collectes ont été effectuées à un poids moyen de $60 \mathrm{~kg}$ (122 jours après le début de l'expérience les porcs ont été abattus).

L'échantillonnage et les analyses des aliments et des excréta ont été réalisés suivant les techniques rapportées par HENRY et RÉRAT (I966); on a calculé individuellement les coefficients d'utilisation digestive apparente de la matière organique (CUDMO) (1) et de l'azote (CUDN) ( $\left.{ }^{2}\right)$ puis, le coefficient de rétention azotée (CRN) $\left({ }^{3}\right)$.

\section{RÉSULTATS}

\section{A. - Expérience en lots}

Les résultats de croissance et d'efficacité alimentaire relatifs à la période de 25 à $60 \mathrm{~kg}$ ne permettent pas de mettre en évidence des différences significatives entre les régimes (tab1. 5). Seules les données concernant la durée totale de l'en-

TABLEAU 5

Performances zootechniques des animaux de 25 à $60 \mathrm{~kg}$

\begin{tabular}{|c|c|c|c|c|c|c|}
\hline \multirow{2}{*}{$-\frac{\text { Régimes }}{\text { Supplément DL-méthionine }}$} & \multicolumn{2}{|c|}{ Soja } & \multicolumn{2}{|c|}{ Levure sulfitique } & \multicolumn{2}{|c|}{ Lactosérum levuré } \\
\hline & 0 & + & 0 & + & 0 & + \\
\hline Gain moyen/j (g) & & & & & & \\
\hline Mâles ......... & 482 & 472 & 469 & 480 & 466 & 449 \\
\hline Femelles $\ldots \ldots \ldots \ldots \ldots \ldots$ & 479 & 486 & 463 & $4_{4} 83$ & 499 & 446 \\
\hline $\begin{array}{c}\text { Moyenne } \ldots \ldots \ldots \ldots \ldots \ldots \\
\text { Consommation } M S / j(\mathrm{~kg})\end{array}$ & 480 & 479 & 464 & 482 & 483 & 448 \\
\hline Mâles $\ldots \ldots \ldots \ldots \ldots \ldots \ldots \ldots$ & 1,60 & 1,58 & 1,58 & 1,51 & 1,59 & 1,55 \\
\hline Femelles $\ldots \ldots \ldots \ldots \ldots \ldots \ldots$ & 1,55 & 1,54 & 1,49 & 1,51 & 1,55 & 1,48 \\
\hline $\begin{array}{c}\text { Moyenne } \ldots \ldots \ldots \ldots \ldots \ldots \\
\text { Indice de consommation } \\
(\mathrm{kg} \text { MS } / \mathrm{kg} \text { de gain) }\end{array}$ & 1,57 & 1,56 & 1,54 & 1,51 & 1,57 & 1,51 \\
\hline Mâles $\ldots \ldots \ldots \ldots \ldots \ldots \ldots$ & 3,32 & $3,3 \prime$ & 3,40 & 3,17 & 3,49 & 3,45 \\
\hline Femelles $\ldots . \ldots \ldots \ldots \ldots \ldots$ & 3,27 & 3,17 & 3,23 & 3,14 & 3,12 & $3,3 / \mathbf{k}$ \\
\hline Moyenne $\ldots \ldots \ldots \ldots \ldots$ & 3,30 & 3,26 & 3,32 & 3,15 & 3,27 & 3,39 \\
\hline
\end{tabular}

Signification statistique $=$ nulle.

(1) CUDMO $=\frac{\text { matière organique ingérée }- \text { matière organique fécale }}{\text { matière organique ingérée }} \times$ roo.

(2) CUDN $=\frac{\mathrm{N} \text { ingéré - } \mathrm{N} \text { fécal }}{\mathrm{N} \text { ingéré }} \times$ 100.

(3) $\quad \mathrm{CRN}=\frac{\mathrm{N} \text { ingéré }-\mathrm{N} \text { fécal }-\mathrm{N} \text { urinaire }}{\mathrm{N} \text { ingéré }-\mathrm{N} \text { fécal }} \times$ roo. 
graissement de 25 à $90 \mathrm{~kg}$ présentent des différences significatives car les variations individuelles sont atténuées (tabl. 6).

Pour tenter de mieux mettre en évidence les différences d'efficacité alimentaire, nous avons calculé les indices de consommation corrigés en tenant compte des différences de durée d'engraissement, donc de besoin d'entretien, suivant la méthode

TABLEAU 6

Performances zootechniques des animaux de 25 à $90 \mathrm{~kg}$

\begin{tabular}{|c|c|c|c|c|c|c|}
\hline Régimes & \multicolumn{2}{|c|}{ Soja } & \multicolumn{2}{|c|}{ Levure sulfitique } & \multicolumn{2}{|c|}{ Lactosérum levuré } \\
\hline Supplément DL-méthionine & 0 & + & 0 & + & 0 & + \\
\hline Gain moyen/j (g) & & & & & & \\
\hline Mâles $\ldots \ldots \ldots \ldots \ldots \ldots$ & 553 & 548 & 539 & 550 & 539 & 533 \\
\hline Femelles $\ldots \ldots \ldots \ldots \ldots \ldots$ & 555 & 575 & 547 & 569 & 567 & 511 \\
\hline $\begin{array}{l}\text { Moyenne }(\mathbf{1}) \ldots \ldots \ldots \ldots \ldots \\
\text { Consommation } M S / j(\mathbf{k g})\end{array}$ & $55^{a}$ & $561^{a}$ & $543^{a b}$ & $560^{a}$ & $553^{a}$ & $522^{b}$ \\
\hline Mâles $\ldots \ldots \ldots \ldots \ldots \ldots \ldots$ & 1,99 & 1,98 & 1,94 & 1,89 & 1,95 & 1,96 \\
\hline Femelles $\ldots \ldots \ldots \ldots \ldots \ldots \ldots$ & 1,94 & 1,90 & 1,91 & 1,91 & 1,96 & 1,86 \\
\hline $\begin{array}{c}\text { Moyenne }\left({ }^{1}\right) \ldots \ldots \ldots \ldots \ldots \\
\text { Indice de consommation } \\
(\mathrm{kg} \text { MS } / \mathrm{kg} \text { gain) }\end{array}$ & $1,97^{a}$ & $1,9 \mathbf{1}^{a}$ & $1,92^{a b}$ & $1,90^{b}$ & $1,95^{a}$ & $1,91^{a b}$ \\
\hline Mâles $\ldots \ldots \ldots \ldots \ldots \ldots \ldots$ & 3,59 & 3,62 & 3,60 & 3,45 & 3,62 & 3,67 \\
\hline Femelles $\ldots \ldots \ldots \ldots \ldots \ldots \ldots$ & 3,49 & 3,30 & 3,50 & 3,37 & 3,46 & 3,67 \\
\hline Moyenne ${ }^{(1)} \ldots \ldots \ldots \ldots \ldots$ & $3,54^{a b}$ & $3,46^{a}$ & $3,55^{a b}$ & $3,41^{a}$ & $3,5_{4}^{a b}$ & $3,67^{b}$ \\
\hline
\end{tabular}

(1) Signification statistique. Classement des moyennes (DUNCAN, 1955). Les valeurs affectées de la même lettre ne sont pas significativement différentes.

proposée par R. FÉVRIER (I952). Les résultats exprimés en pourcentage de l'efficacité du lot soja témoin (S) ont été rapportés dans la figure $I$, en fonction du taux d'acides aminés soufrés du régime.

Les résultats de composition corporelle exprimés par l'analyse des critères habituels sont consignés au tableau 7 .

a) Comparaison des régimes non supplémentés.

Les trois lots non supplémentés présentent des vitesses de croissance semblables, expliquées par des quantités consommées identiques, 1'indice de consommation ne variant pas. A l'abattage, les carcasses présentent des caractéristiques très voisines.

\section{b) Effet de l'addition de DL-méthionine.}

L'addition de méthionine au régime témoin "tourteau de soja " ne modifie pas les performances des animaux ; tout au plus, peut-on déceler une faible amélioration, non significative, de l'efficacité alimentaire pour les femelles. 
Dans le cas de la levure sulfitique, les différences sont un peu plus marquées mais elles n'atteignent pas non plus, le seuil de signification statistique. A l'inverse du cas précédent, l'amélioration porte aussi bien sur les femelles que sur les mâles castrés.

Enfin, l'addition de méthionine au régime " lactosérum levuré " entraîne un effet significativement dépressif sur la vitesse de croissance et sur l'efficacité alimen-

TABLEAU 7

Composition des carcasses

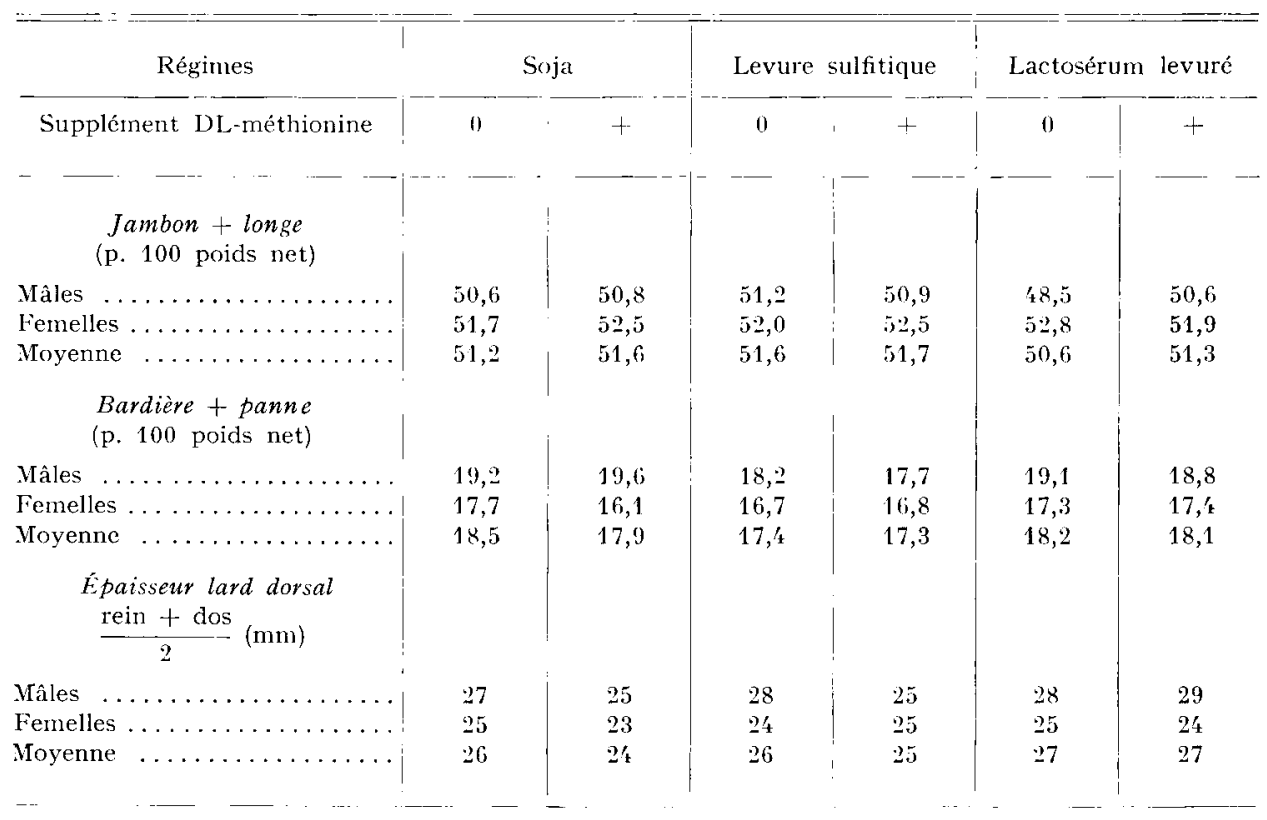

Signification statistique $=$ nulle.

taire, en rapport avec une diminution de la consommation quotidienne. Ce résultat est plus net pour les femelles que pour les mâles castrés, bien que l'interaction sexe-régime ne soit pas significative.

L'addition de méthionine n'a eu, par contre, aucun effet notable sur la qualité des carcasses quelle que soit la nature de la source azotée.

c) Comparaison des régimes supplémentés; effet du taux d'acides aminés soufrés.

Le rapprochement des résultats des trois régimes supplémentés en méthionine permet de comparer trois niveaux d'acides aminés soufrés. Avec un taux de $0,54 \mathrm{p}$. Ioo du régime, le lot levure sulfitique supplémenté ( $A M)$ révèle une efficacité alimentaire corrigée de $4 \mathrm{p}$. Ioo supérieure à celle du lot témoin soja (fig. I ). Lorsque ce dernier est supplémenté (SM) pour atteindre $0,5^{8} \mathrm{p}$. Ioo, l'efficacité est de 5 p. Ioo 
supérieure pour les femelles, et de I,5 p. Ioo inférieure pour les mâles castrés, mais cette interaction n'est pas significative. Enfin pour le taux de 0,6 I p. Ioo atteint avec le régime lactosérum levuré $(\mathrm{BM})$, l'efficacité alimentaire diminue aussi chez les femelles.

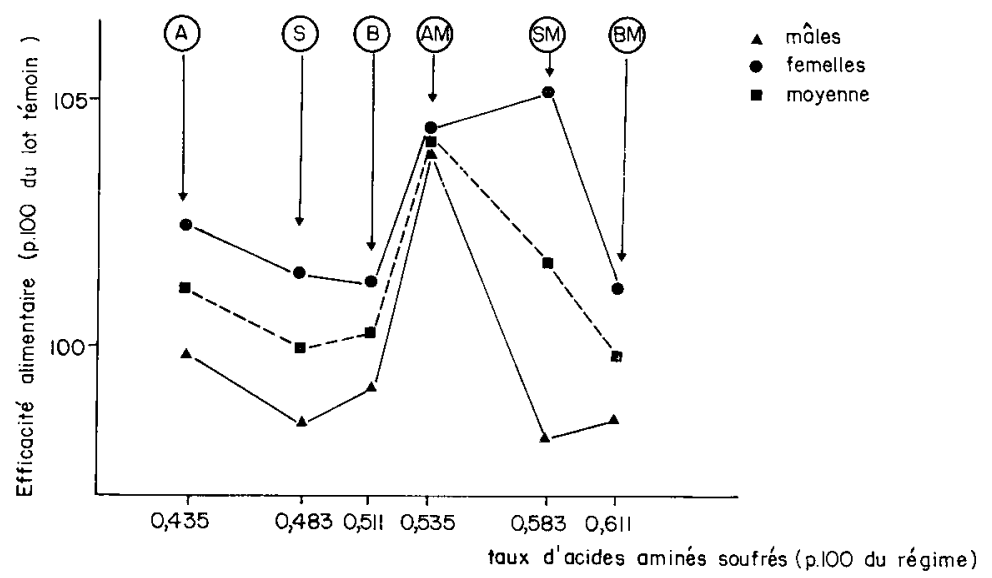

FIG. I. - Efficacité alimentaive

(calculée en pourcentage de l'efficacité du régime soja non supplémenté en fonction du taux d'acides aminés soufrés du régime)
A. Levure sulfitique
AM. Levure sulfitique + o, I p. Ioo DL-méthionine
S. Tourteau de soja
SM. Tourteau de soja +0, I p. Ioo DL-méthionine
B. Lactosérum levuré
BM. Lactosérum levuré + o, I p. Ioo DL-méthionine

\section{B. - Bilan azoté effectué avec le régime "levure sulfitique "}

I a vitesse de croissance des animaux qui a été faible, du fait des changements de logement, n'a pas été influencée par l'addition de méthionine (tabl. 8).

L'utilisation digestive de la matière sèche et de la matière organique ne varie ni avec l'addition de méthionine, ni avec l'âge des animaux. En revanche, l'utilisation digestive de l'azote, si elle n'est pas influencée par l'addition de méthionine augmente de façon hautement significative avec l'âge des animaux.

Au cours des deux collectes de la période de croissance, à $35 \mathrm{~kg}$ environ, la supplémentation en méthionine s'accompagne d'une élévation du coefficient de rétention azotée qui passe de 55,7 à 63,8 p. Ioo. Par contre, au cours des deux dernières collectes, le coefficient de rétention azotée diminue significativement et l'addition de méthionine demeure sans effet.

\section{DISCUSSION}

\section{A. - Comparaison des régimes non supplémentés}

De nombreux auteurs ont déjà établi la bonne efficacité des protéines de levures pour le porc en croissance (LEROY et FF́́VRIER, I947; R. FÉVRIER et al, I952, I954, I955, I957 ; GLAPS et RuszczyC, I955 ; Ruszczyc et GraPS, I955 $a$ et $l$. 
I959; Palamaru et al., I966; Bokorov, Delić et Sreckovic, I963; Csoka, I955 NAvRatil, et SIMENCEK, I966), mais les expériences incluant des comparaisons de protéines de levures à celles du tourteat de soja sont rares. Cependant, les résultats d'Ewan et al. (I969), montrent que le remplacement de protéines isolées de soja par des levures sulfitiques dans le régime de porcelets sevrés avant trois semaines améliore leur vitesse de croissance sans modifier l'indice de consommation.

\section{TABLEAU 8}

Résultats du bilan effectué avec le régime levure sulfitique (*) Utilisation des régimes

\begin{tabular}{|c|c|c|c|c|}
\hline Période & 1 & 2 & 3 & 4 \\
\hline Nombre d'animaux par lot $\ldots . \ldots \ldots$ & 4 & 4 & 3 & 3 \\
\hline Poids moyen $(\mathrm{kg}) \ldots \ldots$. & 33,9 & 36,2 & 58,4 & 62,5 \\
\hline CUDMo $\left\{\begin{array}{l}\text { Levure } \ldots \ldots \ldots \ldots \ldots \\
\text { Levure }+ \text { méthionine } \ldots \\
\text { Moyenne } \ldots \ldots \ldots \ldots \ldots\end{array}\right.$ & $\begin{array}{l}82,1 \\
82,9 \\
82,3\end{array}$ & $\begin{array}{l}80,8 \\
81,8 \\
81,1\end{array}$ & $\begin{array}{l}83,0 \\
82,6 \\
82,9\end{array}$ & $\begin{array}{l}82,7 \\
82,1 \\
82,5\end{array}$ \\
\hline $\operatorname{CUDN}\left\{\begin{array}{l}\text { Levure } \ldots \ldots \ldots \ldots \ldots \\
\text { Levure }+ \text { méthionine } \ldots \\
\text { Moyenne } \ldots \ldots \ldots \ldots\end{array}\right.$ & $\begin{array}{l}79,3 \\
79,2 \\
79,3^{a}\end{array}$ & $\begin{array}{l}81,1 \\
80,2 \\
80,7 a\end{array}$ & $\begin{array}{l}83,2 \\
83,3 \\
83,2^{b}\end{array}$ & $\begin{array}{l}83,3 \\
83,2 \\
83,3^{b}\end{array}$ \\
\hline$\left\{\begin{array}{l}\text { Levure } \ldots \ldots \ldots \ldots \ldots \\
\text { Levure }+ \text { méthionine } \ldots \\
\text { Moyenne } \ldots \ldots \ldots \ldots \ldots\end{array}\right.$ & $\begin{array}{l}5 \cdot 4,1 x \\
62,4^{y} \\
58,2^{a}\end{array}$ & $\begin{array}{l}57,4^{x} \\
65,2^{y} \\
61,3^{a}\end{array}$ & $\begin{array}{l}48,6 \\
49,3 \\
49,1^{0}\end{array}$ & $\begin{array}{l}48,3 \\
47,6 \\
48,0^{b}\end{array}$ \\
\hline
\end{tabular}

Signification statistique $=$ Effet période (exposants $a$ et $b$ ) CUDN, CRN : hautement significatif $(\mathrm{P}<0,01)$

Effet méthionine (exposants $x$ et $y$ )

CRN : significatif $(\mathrm{P}<0,05)$

* Vitesse de croissance au cours de l'essai (122 jours) $=$ levure : ${ }_{4} 41 \mathrm{~g} / \mathrm{j}$. Levure + méthionine : ${ }_{4} 29 \mathrm{~g} / \mathrm{j}$.

Dans la présente étude, les levures permettent les mêmes performances que le tourteau de soja, alors que nous avions enregistré en cages à bilan, des résultats inférieurs avec les mêmes régimes (ColOMER-RochER et FÉVRIER, I968). De plus, la diminution du coefficient de rétention dans 1'actuel bilan azoté (I5 p. IOo) est nettement moins importante que précédemment (30 p. I0o) entre 35 et $60 \mathrm{~kg}$ de poids vif.

Ces deux contradictions apparentes peuvent s'expliquer par les différences des niveaux alimentaires imposés au cours des diverses expériences (tab1. 4). I1 paraît probable que les conditions antérieures de rationnement contraignaient les animaux à utiliser les protéines à des fins énergétiques.

Or, une part importante de l'azote des levures provient des acides nucléiques. L'azote purique représente chez Torula utilis, selon le milieu de culture, 9,8 à 
24,2 p. Ioo de l'azote total (EDDY, I958), que l'animal excrète dans l'urine sous forme d'allantoïne, en pure perte pour la synthèse protéique et le métabolisme énergétique (TERROINE, I960).

Par ailleurs, l'expérience présente montre que la bonne utilisation des levure, passe par une adaptation digestive de l'animal, résultat déjà obtenu précédemment (Colomer-Rocher et FÉvrier, I968).

Le régime "tourteau de soja " bien qu'également pauvre en azote total est en réalité plus riche que les autres en éléments protidiques digestibles. Aussi, n'est-il pas étonnant qu'il donne les meilleurs résultats lors d'une restriction alimentaire trop sévère.

\section{B. - Effets de la supplémentation des régimes}

Nos résultats permettent de conclure à la déficience en acides aminés soufrés d'un régime orge-levure sulfitique à $\mathrm{I} 3 \mathrm{p}$. Ioo de protéines pour le porc de 35 à $40 \mathrm{~kg}$, en accord avec les recommandations de RÉRAT et LOUGNON (I966). Le déficit ne semble pas exister chez les animaux plus lourds et la supplémentation ne présente de ce fait, que peu d'intérêt pour la vitesse de croissance, sur l'ensemble de la durée d'engraissement. Ces observations confirment celles de NAVRaTIL et SIMENCEK (I966) sur la supplémentation en méthionine d'un régime : céréales-tourteau d'arachidelevure.

En revanche, l'inappétence de la ration orge-lactosérum levuré supplémenté, ainsi qu'une baisse régulière de l'efficacité alimentaire lorsque le taux d'acides aminés soufrés dans la ration passe de 0,54 à 0,58 , puis à 0,6 I p. Ioo (fig. I), suggèrent un effet légèrement toxique de la ILL-méthionine (RÉRAT et al., I962). Les acides aminés soufrés ne représenteraient donc que le facteur limitant secondaire du régime, et la simple connaissance des teneurs en acides aminés essentiels ne permet pas d'en déterminer le facteur limitant primaire. Avec le même régime nous avons obtenu un résultat identique chez le Rat qui, de plus, ne répond pas à la supplémentation en L-lysine (CoLOMER-Rocher et FÉvrier I97I).

L'absence de la donnée de rétention azotée dans le cas du régime témoin orgesoja, ne nous permet pas de conclure à sa déficience en acides aminés soufrés, bien que le besoin ne soit pas couvert. L'am élioration significative de l'efficacité alimentaire observée chez les femelles est toutefois en accord avec les résultats de LougNoN (I970) obtenus pour un régime comparable (orge-blé-soja), et avec ceux de RÉRAT et HeNRY (I970), qui recommandent un apport plus important d'acides aminés soufrés chez les femelles que chez les mâles castrés.

\section{CONCLUSION}

En définitive, la comparaison des protéines de levure sulfitique et de lactosérum levuré à celles du tourteau de soja, chez le Porc en croissance, nous permet de préciser trois points essentiels.

$\mathrm{I}^{\circ}$ Un niveau alimentaire trop bas en début de croissance met en évidence un inconvénient de l'utilisation des levures, que nous attribuons à leur azote nucléique, et aux difficultés de digestion de leurs protéines par le jeune animal. 
$2^{\circ}$ Lorsque le niveau alimentaire devient suffisant, même si on rationne encore les animaux, la valeur biologique des levures, mesurée par la vitesse de croissance ou l'efficacité alimentaire, devient équivalente à celle du tourteau de soja.

$3^{\circ}$ Un régime à base de lactosérum levuré renfermant I3 p. Ioo de protéines, qui présente un équilibre en acides aminés meilleur qu'une ration isoprotidique à base de tourteau de soja ne nécessite pas 1'addition de méthionine. En revanche, pour une même teneur en protéines, la supplémentation d'un régime orge-levure sulfitique en acides aminés soufrés se révèle intéressante chez le jeune porc $(25-60 \mathrm{~kg})$.

Une qualité essentielle des levures tient à leur haute teneur en lysine remarquablement stable aux traitements thermiques (ADRIAN et FrangNE, I970). Elle explique la réponse positive à la supplémentation en méthionine de notre régime, alors qu'avec l'incorporation de soja, la lysine devient le facteur limitant primaire et 1'addition d'acides aminés soufrés reste quasi inefficace. Cette particularité conduirait à recommander, à digestibilité égale, les protéines de levure plutôt que celles de soja dans la ration du porcelet.

Reçu pour publication en octobre 1972.

\section{SUMMARY}

COMPARISON BETWEEN THE PROTEIN VALUE OF SUI.PHITE YEAST

AND OF YEASTED WHEY IN GROWING-FINISHING PIG DIETS BASED UPON BARLEY. EFFECT OF SUPPIEMENTATION BY DL-METHIONINE

Seventy-two Large White pigs between 25 and $90 \mathrm{~kg}$ were used to compare the efficiency of 3 protein sources : sulphite yeast, yeasted-whey and soybean oil-meal " 44 " dicts based upon barley and isoproteic (I3 p. I $00 \mathrm{~N} \times 6.25$ ), isolaloric $(4.300 \mathrm{kcal} / \mathrm{kg}$ ) and isocellulosic (ro p. 100 crude fiber), supplemented or not by o. I p. I oo synthetic DL-methionine. The non supplemented diets led to performances identical for all the animals. The favourable effect of supplementation did not reach the threshold of statistical significance in the diets containing sulphite yeast and soybean oil-meal. On the other hand, methionine supplementation of the yeasted whey diet led to significant reduction of the growth rate probably due to an excess of sulphur amino acids. In contrast, the nitrogen retention coefficient was significantly improved by sulphur amino acid supplementation of the diets containing sulphite yeast. Finally, the carcass quality remained constant whatever the source of protein or sulphur amino acid level of the diet.

These findings show that in the present experimental conditions, sulphite yeast and yeasted whey could without difficulty replace soybean oil-meal in the growing-finishing Pig rations.

\section{RÉFÉRENCES BIBLIOGRAPHIQUES}

Adrian J., Francise R., ig7o. Valeur protidique de la levure Torula en fonction des modalités thermiques du chauffage. Ind. Alim. agr., 87, 393-399.

Bokorov T., IElic I., SReckovic A., I963. Comparison of the biological value of fooder yeast (Torula utilis), dried milk and fishmeal as additives to gluten feed and soya bean oil for fattening pigs (serbo-croate). Stocarstvo, 17, 560-565.

Calkt C., Adrian J., Jacovot R., ig62. Étude de la valeur nutritionnelle de la levure utilisée sous trois formes différentes : levure sèche, levure autolysée et autolysat de levure. Ann. Zootech., 11, Io3-I I4.

Cochran W. O., Cox G. M., 1957. Experimental desings, 6ix p. Ed. John Wiley and sons., New York.

Colomer-Rocher F., Fevrifi C., I968. Valeur alimentaire des protéines de la levure sulfitique et du lactosérum levuré chez le porc en croissance. Ann. Zootech., 17, 409-427. 
Colomer-Rocher F., Février C., I97I. Valeur alimentaire de la levure sulfitique (TORUTIL) et du lactosérum levuré (S. A. V.) pour le Rat : effet de la supplémentation par la lysine et la méthionine. Ann. Zootech., 20, 91-105.

Csoka S., I965. Effect of different amounts of dried brewer's yeast in feed mixtures on the fattening performances of hungarian Yorkshire pigs fed from self feeders (Hong.). Allattenyesztes, 14, 55-66.

Duncan D. B., 1955. Multiple range and multiple F tests. Biometrics, 11, r-42.

Evdy A. A., 1958. Aspects of the chemical composition of yeast in Cook A. H. The chemistry and biology of yeasts, New York, Academic Press.

Ewan R. C., Wastell M. E., Bicknell E. J., Speer V. C., ig69. Performance and deficiency symptoms of young pigs fed diets low in vitamin E and selenium. J. Anim. Sci., 29, 912-915.

Février R., r952. L'indice de consommation est-il, chez le Porc, le témoin fidèle de l'efficacité d'une ration? Ann. Zootech., 1, I75-I84.

Fŕvrier R., I954. Valeur alimentaire des levures. II. Influence du mode de séchage de quelques levures de distillerie. Ann. Zootech., 3, 219-222.

FÉvrier R., VACHel J.-M., 1955. Valeur alimentaire des levures. III. Comparaison de trois levures d'origines différentes. Ann. Zootech., 4, I 29-13I.

FÉvrier R., Boccard R., I957. Valeur alimentaire des levures. IV. La levure séchée sur marc de pommes. Ann. Zootech., 6, 269-28I.

FÉvrier R., Leroy A.-M., Roche J., I952. Influence du mode de séchage sur la valeur alimentaire de la levure de distillerie. Ann. Zootech., 1, I-Io.

GLAPS J., RuSzcyc Z., I955. Studies on the use of fodder yeast and maize in the fattening of pigs (pol.). Rocz, Nauk. Rol. (B), \%0, I39-152.

Henry Y., Rérat A., I966. Utilisation des pommes de terre déshydratées et fraîches dans l'alimentation du porc en croissance en comparaison avec l'orge. Ann. Zootech., 15, 23 I-25I.

Leroy A.-M., FÉvrier R., I947. Emploi de la levure de distillerie pour l'alimentation des animaux domestiques. Ann. agron. Paris, 4, $578-596$.

Lougnon J., I97o. Supplémentation par la lysine et la méthionine d'aliments chez le porc en croissancefinition : influence du sexe et du rationnement. Journées Rech. Porcine en France, Paris, 85-90, I. T. P. éditeur.

Navratil. B., S̈mpincek K., I966. Comparison of the effectiveness of dried feed yeasts supplemented, with L-lysine and DL-méthionine and fish meal in the fattening of pigs (tchèque). Zivoc. $V y r ., 11$, I 7 I-I 82.

Palamaru E., Gondos M., Geamau N., Sirbu M., Petrovszky R., Romanovchi S., Hagea I., Covaliu C., ERdei M., Masim V., Stavri J., rg66. Efficacité do la levure fourragère administrée dans la nourriture des jetnes porcs et des jeunes volailles (roumain). Lucr. Inst. Cercer. Zoot., 13, I 85-20I,

Pion R., I967. Données non publiées.

Pion R., Fauconneau G., ig66. Les acides aminés des protéines alimentaires. Méthodes de dosage et résultats obtenus. Cahier A. E. C., 6, r 55-175, A. E. C., Commentry.

Rérat A., Henry Y., I964. Étude du besoin azoté chez le Porc en croissance. I. Utilisation de la farine de poisson à trois taux différents. Ann. Zootech., 13, 5-34.

RÉrat A., HENRY Y., I97o, Étude du besoin en acides aminés soufrés du porc en croissance. Journées Rech. Porcine en France, Paris, 61-71, I. T. P. éditeur.

Rérat A., Lougnon J., 1966. Les besoins en aminoacides du porc en croissance, Cahier A.E.C., 6, $34 \mathrm{I}-422$, A. E. C., Commentry.

Rérat A., Lougnon J., Pion R., I962. Supplémentation d'un régime complexe par la DL-méthionine de synthèse chez le Porc. Ann. Zootech., 11, I59-172.

Rous J., Petkovs S., Stefk M., Skrivan M., r965. Dried fodder yeast (Torulopsis utilis) and DL-methionine in feed rations for chicks (tchèque). Zivoc. Vyr., 10, 749-758.

Ruszczyc Z., GLaps J., I955 a. The use of yeast slop (fresh brewery yeast) for fattening pigs (pol.). Rocz. Nauk. Rol. (B), 70, 153-167.

Ruszcyc Z., Glaps J., r955 b. The use of postsulphite yeast in the fattening of pigs (pol.). Rocz. Nauk. Rol. (B), 70, I69-180.

RuszczY'C Z., Glaps J., I959. The feeding value of yeast produced on postsulphite lyes and postsulphite wash for fattening pig (pol.). Rocz. Nauk. Rol. (B), 74, I37-I 44

Terroine E. F., I960. Le métabolisme nucléique. Éd. C. N. R. S., Paris. 